\title{
Inhibition of mammalian target of rapamycin complex 1 signaling by $n-3$ polyunsaturated fatty acids promotes locomotor recovery after spinal cord injury
}

\author{
JIPING NIE ${ }^{1,2 *}$, JIAN CHEN $^{3 *}$, JIANGUO YANG $^{4 *}$, QINQIN PEI $^{3 *}$, JING LI $^{3}$, JIA LIU $^{5}$, LIXIN XU $^{3}$,

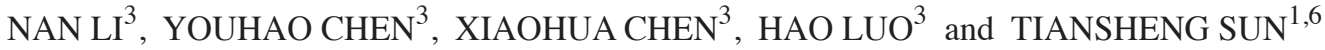 \\ ${ }^{1}$ Southern Medical University, Guangzhou, Guangdong 510515; ${ }^{2}$ Department of Orthopedics,
}

The Second Affiliated Hospital of Inner Mongolia Medical University, Huhhot, Inner Mongolia Autonomous Region 010050; ${ }^{3}$ Department of Orthopedics, Three Gorges Central Hospital of Chongqing, Chongqing 404000; ${ }^{4}$ Department of Orthopedics, Huhhot First Hospital, Huhhot, Inner Mongolia Autonomous Region 010020; ${ }^{5}$ Department of Orthopedics, Affiliated Hospital of Youjiang Medical College for Nationalities, Baise, Guangxi 533000; ${ }^{6}$ Department of Orthopedics, Beijing Army General Hospital, Beijing 100700, P.R. China

Received March 14, 2017; Accepted January 9, 2018

DOI: $10.3892 / \mathrm{mmr} .2018 .8583$

\begin{abstract}
The present study aimed to explore the effects of n-3 polyunsaturated fatty acids (PUFAs) on autophagy and their potential for promoting locomotor recovery after spinal cord injury (SCI). Primary neurons were isolated and cultured. Sprague-Dawley rats were randomly divided into three groups and fed diets with different amounts of n-3 PUFAs. A model of spinal cord contusion was created at the T10 spinal segment and the composition of PUFAs was analyzed using gas chromatography. Spinal repair and motor function were evaluated postoperatively. Assessment of the effects of n-3 PUFAs on autophagy and mammalian target of rapamycin complex 1 (mTORC1) was performed using immunofluorescence staining and western blotting. In vitro, n-3 PUFAs inhibited mTORC1 and enhanced autophagy. The n-3 PUFA levels and the ratio of n-3 PUFA to n-6 PUFA in the spinal cord and serum of rats fed a high-n-3 PUFA diet were higher before and after operation $(\mathrm{P}<0.05)$. Additionally, rats in the high-n-3 PUFA group showed improved motor function recovery, spinal cord repair-related protein expression level (MBP, Galc and GFAP).
\end{abstract}

Correspondence to: Professor Tiansheng Sun, Department of Orthopedics, Beijing Army General Hospital, 5 Nanmengcang, Beijing 100700, P.R. China

E-mail:2381049996@qq.com

Professor Hao Luo, Department of Orthopedics, Three Gorges Central Hospital of Chongqing, 165 New City Road, Chongqing 404000, P.R. China

E-mail: yueer327@126.com

*Contributed equally

Key words: polyunsaturated fatty acids, spinal cord injury, mammalian target of rapamycin, autophagy, locomotor recovery
Expression levels if these protiens in the high-n-3 PUFA diet group expressed the highest levels, followed by the low-n-3 PUFA diet group and finally the control group $(\mathrm{P}<0.05)$. high-n-3 PUFA diet promoted autophagy ability and inhibited activity of the mTORC1 signaling pathway compared with the low-n-3 PUFA diet group or the control group $(\mathrm{P}<0.05)$. These results suggest that exogenous dietary n-3 PUFAs can inhibit mTORC1 signaling and enhance autophagy, promoting functional recovery of rats with SCI.

\section{Introduction}

Restoring normal physiological function after traumatic spinal cord injury (SCI) is difficult under conditions of oxygen and energy deprivation, due to the severely compromised energy metabolism in the injured spinal cord. Furthermore, new nerves cannot grow because of the limited regenerative ability of the central nervous system at the sites of injury, leading to a loss of sensorimotor function below the point of injury $(1,2)$. Various measures to repair SCI have been developed; these can, to a certain extent, promote axonal regeneration and functional recovery, but there is still a long way to go to achieve complete repair (3-8).

Polyunsaturated fatty acids (PUFAs) are essential for mammals, but n-3 PUFAs cannot be synthesized by the human body and must be obtained from foods. Research has indicated that n-3 PUFAs are closely involved in the physiological activities of the nervous and immune systems, among others (9-13). Investigations have revealed that n-3 PUFAs play an important role in regulating the mammalian target of rapamycin complex 1 (mTORC1) signaling pathway in the body (14-16).

Autophagy is a highly conserved cell degradation process, which involves isolating part of the cytoplasm and organelles in a bilayer vesicle and delivering them to lysosomes for degradation, eventually recycling the large decomposed molecules; LC3-II is one of the markers of autophagy $(17,18)$. Autophagy activity is widespread in injured spinal cords, and studies have 
shown that autophagy is significantly activated in the days just after SCI (19-22). Activation of autophagy can clear intracellular damaged proteins and protect against neuronal loss to promote recovery of motor function after SCI (23-25). The role of autophagy in the experimental study of SCI is attracting more and more attention.

mTOR is a highly-conserved serine/threonine protein kinase consisting of two different compounds in the body, mTORC1 and mTORC2. Through nutrient, energy and growth factor signaling pathways, mTORC1 regulates cell metabolism, growth, proliferation, survival and autophagy $(26,27)$. Studies have shown that inhibiting mTORC1 can enhance autophagy and help repair injuries (13,28-37). It is known that the mTORC1 signaling pathway and autophagy activity are involved in the repair of articular cartilage and nervous tissues, playing a vital role in the recovery of damaged tissue $(13,15)$, but its role in SCI remains unclear.

In this study, we created a rat SCI model and fed the rats with a diet high in n-3 PUFAs to enhance the content of n-3 PUFAs and the n-3/n-6 PUFA composition, and then explored whether n-3 PUFAs can regulate autophagy through the mTORC1 signaling pathway to promote repair of SCI.

\section{Materials and methods}

In vitro experiments. Primary Schwann cells were obtained from National Infrastructure of Cell Line Resource (RSC-96, 3111C0001CCC000664; Beijing, China) and cultured according to previously-published protocols (38). mTORC1 is a signaling pathway sensitive to amino acids (aa). Removal and readdition of aa eliminates and stimulates mTORC1 activity, respectively. Thus, aa starvation was used in these experiments. Exogenous docosahexaenoic acid (DHA) and arachidonic acid (AA) were used as a representative n-3 PUFA and n-6 PUFA, respectively. Neuronal cells were starved of amino acids by culturing in Dulbecco's phosphate-buffered saline for $30 \mathrm{~min}$. After this, either DHA $(50 \mu \mathrm{M})$ or AA $(50 \mu \mathrm{M})$ (both from Cayman Chemical Company, Ann Arbor, MI, USA) was added, cells were returned to culture for $30 \mathrm{~min}$, then proteins were extracted and the activity of mTORC1 downstream protein p-S6 was analyzed to investigate the influence of PUFAs on mTORC1. To investigate expression of the autophagy-related protein LC3-II (Cell Signaling Technology, Inc., Danvers, MA, USA), cultures were treated in the same way but returned to culture for $3 \mathrm{~h}$ before proteins were extracted to study the influence of n-3 PUFAs on autophagy.

Animal groupings. Animal experiments were approved by the animal experimental ethics committee of Southern Medical University (GuangZhou, China) and all animals were purchased from the Laboratory Animal Centre at Southern Medical University. Adult Sprague-Dawley (SD) rats (90 female, weight: 200-250 g) were randomly divided into three groups: A normal diet group (CON) (n=30), a low n-3/n-6 diet group (Low), and a high n-3/n-6 diet group (High) (Table I).

SCI model. The three groups of rats were fed in separate cages for one week before operation, and continued on the same diet after model establishment. Rats were anesthetized with chloral hydrate (10\% chloral hydrate, $40 \mathrm{mg} / \mathrm{kg}$ ) and placed in the prone position. Laminectomy was performed at level T9-10 to expose the spinal cord. In the CON group, laminectomy was performed without SCI; in the other two groups, SCI was created by the modified Allen method at level T10, and paralysis of both hind legs was successfully achieved. The wound was then sterilized and closed. Rats received intraperitoneal injection of $5 \mathrm{ml}$ saline for rehydration and daily administration of penicillin $(100 \mathrm{mg} / \mathrm{kg}$ ) for 3 days to prevent infection. Manual bladder expression was performed twice a day until re-establishment of the voiding reflex. All operations were performed by the same surgeon to reduce inter-operator variation.

Gas chromatography. Blood samples were collected from the caudal vein, and the rats were killed. A $2-\mathrm{cm}$ long section of the spinal cord at the injured location was quickly removed before and $12 \mathrm{~h}$ after surgery $(\mathrm{n}=6)$. Fatty acids were extracted from serum and the spinal cord and the content was analyzed according to existing published methods $(39,40)$. A Perkin-Elmer Clarus 500 chromatographic analyzer (PerkinElmer, Inc., Waltham, MA, USA) was used for gas chromatographic analysis. The fatty acid composition was identified by comparing the peak time to that of standard specimens (purchased from Sigma-Aldrich; Merck KGaA, Darmstadt, Germany) by gas chromatography.

Behavioral assessment. Hindlimb motor function was assessed using the open-field Basso, Beattie and Bresnahan (BBB) locomotor scoring behavioral assessment system $(41,42)$ with scores ranging from 0 to 21 . Scoring was performed by two observers on the first postoperative day and then weekly up to 8 weeks.

Five rats were selected randomly from each group at week 8 after the operation, anesthetized and placed on a quiet desktop. The head of the electrodes was inserted into the scalp at the motor cortex and the end into the quadriceps muscle. The latency and amplitude of the motor-evoked potential were detected according to the manufacturer's instructions. Measurements were performed three times for each rat.

Reverse transcription-quantitative polymerase chain reaction $(R T-q P C R)$. Spinal cords were harvested at 8 weeks after surgery, and RT-qPCR was performed to determine the relative mRNA levels of MBP, Galc, GFAP and TUBB3 in the spinal cords containing the lesion site $(n=3)$. RNA was extracted using TRIzol and cDNA was synthesized using an RT Reagent kit (Takara Biotechnology Co., Ltd., Dalian, China) according to the manufacturer's instructions. The cDNA concentration was tested using a NanoDrop 2000 spectrophotometer (Thermo Fisher Scientific, Inc., Wilmington, DE, USA) after reverse transcription. According to the instructions of the TaKaRa Perfect Real Time Reagent kit (Takara Biotechnology Co., Ltd.), qPCRs were performed in 0.2-ml thin-walled reaction tubes using an ABI Prism 7500 Sequence Detection System (Applied Biosystems; Thermo Fisher Scientific, Inc., Waltham, MA, USA). The sequences of the primers (Sangon, Shanghai, China) were listed in Table II.

GAPDH was used as an endogenous control to normalize expression levels of the target genes between the different groups. Relative expression of the PCR products was determined using the $\Delta \Delta \mathrm{Cq}$ method. 
Table I. Polyunsaturated fatty acid composition and content in the diets of experimental rats.

\begin{tabular}{lccr}
\hline Type of fatty acids & CON (g/kg diet) & Low n-3/n-6 (g/kg diet) & High n-3/n-6 (g/kg diet) \\
\hline C18:3, n-3, $\alpha$-linoleic acid & 0.41 & 0.55 & 0.41 \\
C20:5, n-3, eicosapentaenoic acid (EPA) & 2.80 & 4.47 & 29.75 \\
C22:5, n-3, docosapentaenoic acid (DPA) & 0.79 & 0.85 & 0.83 \\
C22:6, n-3, docosahexaenoic acid (DHA) & 2.41 & 3.63 & 17.19 \\
n-3, total & 6.41 & 9.50 & 48.18 \\
C18:2, n-6, linoleic acid & 50.31 & 9.75 & 6.43 \\
C20:4, n-6, arachidonic acid (AA) & 3.70 & 0.54 & 0.39 \\
n-6, total & 54.01 & 10.29 & 6.82 \\
n-3/n-6 & 0.118 & 0.923 & 7.04
\end{tabular}

Fatty acid values in the table are from gas chromatography-mass spectrometry (GC-MS). Rats in the CON group were fed normal rat chow.

Table II. Sequences of polymerase chain reaction target genes.

\begin{tabular}{lll}
\hline MBP & 5'-GGCAATGGTGGGACTCAAAA-3' & 5'-GGGACCCGCTCCTTCAAC-3' \\
Galc & 5'-GAGTCCACAACCATCCTTCTGAG-3' & 5'-ACACCAGGCTGCTTGAACAC-3' \\
GFAP & 5'-GCATCGCTTCACACTGCGCC-3' & 5'-ACACACCGCCAGTCCGAGGA-3' \\
TUBB3 & 5'-GCCCAAGTGAAGTTGCTTGC-3' & 5'-TGCCCTGAAGAGCTGGTAG-3' \\
GAPDH & 5'-CCACTCCTCCACCTTTGAC-3' & 5'-ACCCTGTTGCTGTAGCCA-3' \\
\hline
\end{tabular}

Immunofluorescence analysis. Rats were deeply anesthetized with chloral hydrate $12 \mathrm{~h}$ after operation $(\mathrm{n}=3)$, and transcardiac perfusion was performed as previously described (43). A 2-cm-long spinal cord segment centered on the injured area was removed and placed into $4 \%$ paraformaldehyde overnight, and then samples were cryoprotected in $30 \%$ sucrose buffer for cryosectioning. The spinal cords were sectioned into 5- $\mu \mathrm{m}$-thick coronal sections with a cryostat and used for immunofluorescent staining. Each section was washed with phosphate-buffered saline (PBS) and treated with $0.1 \%$ Triton X-100 for 5 min. Sections were then blocked with $10 \%$ bovine serum albumin (BSA) for $1 \mathrm{~h}$, then incubated with primary antibody at $4^{\circ} \mathrm{C}$ overnight as follows: MBP (1:150), Galc (1:100), GFAP (1:100) (Cell Signaling Technology, Inc.). After washing with PBS for $1 \mathrm{~h}$, sections were incubated with the appropriate fluorescent-labeled goat-anti rabbit secondary antibody (Santa Cruz Biotechnology, Inc., Santa Cruz, CA, USA), and then examined under a fluorescence microscope (Nikon, Tokyo, Japan). Specimens were collected from rats killed at $12 \mathrm{~h}, 1$ week and 2 weeks postoperatively and stained with LC3-II (1:50; Cell Signaling Technology, Inc.), and then observed under a fluorescence microscope (Nikon).

Western blotting. The fresh spinal cords of rats killed 2 weeks postoperatively $(n=3)$ were pounded in a mortar to a powder and centrifuged at $14,000 \mathrm{x} \mathrm{g}$ for $15 \mathrm{~min}$ at $4^{\circ} \mathrm{C}$. The supernatant was then collected and boiled for $10 \mathrm{~min}$ at $100^{\circ} \mathrm{C}$. The appropriate amounts of proteins were loaded onto a $10 \%$ sodium dodecyl sulfate polyacrylamide gel and subjected to electrophoresis (SDS-PAGE), then electroblotted onto a polyvinylidene fluoride membrane. After blocking for $1 \mathrm{~h}$ at room temperature in a solution of $5 \%(\mathrm{w} / \mathrm{v})$ skim milk in Tris-buffered saline containing $0.05 \%$ Tween-20, the membrane was incubated with antibodies against the mTOR-related proteins p-S6 (1:100), p-Akt (1:50), p-S6K (1:100), or 4EBP1 (1:100) (all from Cell Signaling Technology, Inc.) at $4^{\circ} \mathrm{C}$ overnight, then incubated with the appropriate secondary goat anti-rabbit antibody (Bioworld, Dublin, $\mathrm{OH}$, USA) after washing the membrane for $1 \mathrm{~h}$ at room temperature. Finally, after exposure and development, the blots were photographed and scanned for analysis.

Statistical analysis. All quantitative data are presented as mean \pm standard error of the mean, and differences among groups were considered significant at $\mathrm{P}<0.05$. Statistical analysis was performed by analysis of variance followed by Bonferroni's post-hoc test (multiple-comparison tests) among three groups, using the statistical analysis software SPSS 13.0 (SPSS, Inc., Chicago, IL, USA).

\section{Results}

PUFAs altered the activity of MTORC1 and the expression of autophagy-related genes in vitro. When the same dose of DHA was added to neurons with decreased p-S6 induced by aa or AA, the p-S6 activities in both the groups decreased (Fig. 1A). After adding the same dose of AA or DHA to the two groups starved of aa, the expression of autophagy protein LC3-II decreased or increased, respectively. The effect on LC3I was opposite to that of LC3-II and the effect on P62 was the same as that of LC3-II (Fig. 1B). Addition of AA or DHA to the neurons provided with aa led to an increase or decrease 
A
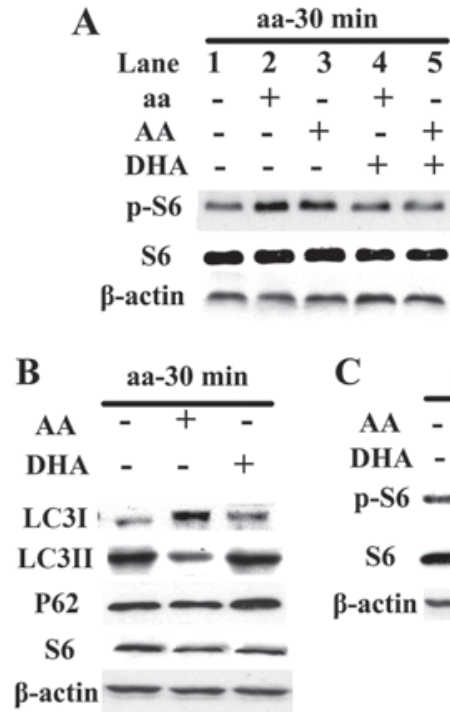

Figure 1. Effects of exogenous polyunsaturated fatty acids (PUFAs) on mammalian target of rapamycin complex 1 (mTORC1) and autophagy. (A) AA activated mTORC1 independently (lane 1 vs. lane 3), while docosahexaenoic acid (DHA) ameliorated amino acid- and arachidonic acid (AA)-induced activation of the mTORC1 signaling pathway (lane 2 vs. lane 4; lane 3 vs. lane 5). (B) Under amino acid starvation, AA and DHA inhibited and enhanced expression of the autophagic marker LC3-II protein, respectively; the effect on LC3I was opposite to that of LC3-II and the effect on P62 was the same as LC3-II. (C) DHA independently inhibited mTORC1.

in p-S6 activity, respectively; neurons given the same dose of DHA and AA simultaneously showed no significant change in p-S6 activity compared with those without treatment (Fig. 1C).

Contents and composition of PUFAs in serum and spinal cord. Gas chromatography was used to analyze the serum and spinal cord preoperatively and postoperatively to study the effects of a high-n-3 PUFA diet on the percentage of PUFAs. The results showed that, preoperatively, the content of n-3 PUFAs increased, while that of n-6 PUFAs decreased, significantly in both the spinal cord and serum of the group fed a high-n-3 PUFA diet compared with those fed a low-n-3 PUFA diet or the control group $(\mathrm{P}<0.05$; Fig. $2 \mathrm{~A}$ and $\mathrm{B})$; the same result was observed postoperatively, $(\mathrm{P}<0.05$; Fig. $2 \mathrm{C}$ and $\mathrm{D})$. The percentages of PUFAs in the serum and spinal cord of each group before operation were lower than those after operation, but the difference was not significant. In summary, a high-n-3/n-6 PUFA diet significantly increased both the n-3 PUFA content and n-3/n-6 PUFA ratio in the serum and spinal cord of rats in vivo.

Assessment of locomotor behavior. Complete paralysis of the hind legs after surgery and a BBB score of 0 indicated successful establishment of the SCI model. BBB scores revealed gradual restoration of movement of rats in each group by 8 weeks after surgery. Compared with the group fed a low-n-3 PUFA diet and the control group at each time-point, BBB scores of the rats in the high-n-3 PUFA diet group were higher, but the group that received n-3 PUFA lavage had much higher scores $(\mathrm{P}<0.05$; Fig. 3A). Rats in the high-n-3 PUFA diet group showed shorter latency times of motor evoked potential $(5.3 \pm 0.32 \mathrm{msec})$ and higher amplitude $(60.30 \pm 4.72 \mathrm{msec})$ than those in the low-n-3 PUFA diet group $(6.19 \pm 0.42$ and $33.19 \pm 4.49 \mathrm{msec}$, respectively) or the control group $(9.51 \pm 0.39$ and $31.61 \pm 4.22 \mathrm{msec}$, respectively), and all differences were statistically significant $(\mathrm{P}<0.05$; Fig. 3B-D). These results indicate that the high-n-3/n-6 PUFA diet was more effective in promoting functional recovery.

Changes in tissue repair related proteins and $m R N A$ expression after operation. The results of immunofluorescence staining showed that all three groups had the same patterns of expression of MBP, Galc and GFAP at the injured sites: the high-n-3 PUFA diet group expressed the highest levels, followed by the low-n-3 PUFA diet group and finally the control group (Fig. 4A); the differences were statistically significant $(\mathrm{P}<0.05)$ (Fig. 4 B-D). The results of RT-PCR were in agreement with immunofluorescent staining, showing the same trends, with statistically significant differences $(\mathrm{P}<0.05)$ (Fig. 5). These results indicate that a diet high in $n-3 / n-6$ PUFAs can significantly increase MBP, Galc, GFAP and TUBB3 mRNA expression in injured spinal cords.

A diet high in n-3PUFAs promoted autophagy. No significant difference in LC3-II expression was found among the three groups, as shown by the autophagy protein test performed $12 \mathrm{~h}$ postoperatively (Fig. 6A and B). However, expression of the LC3-II autophagy protein was significantly higher in the high-n-3 PUFA diet group at 1 and 2 weeks after operation compared to the low-n-3 PUFA diet group or the control group $(\mathrm{P}<0.05$; Fig. 6A, C and D). Thus, a high n-3/n-6 PUFA diet promoted LC3-II expression in the injured spinal cords.

A diet high in n-3 PUFAs inhibited activity of the mTORC1 signaling pathway. The high-n-3 PUFA diet group showed lower activities of p-Akt (S473), p-S6 (S235/S236), p-S6 (S240/S244), and p-S6K in the spinal cords at 2 weeks after surgery compared to the low-n-3 PUFA diet group or the control group (Fig. 7A), and comparison of the density of the bands produced by western blotting showed that the difference was statistically significant $(\mathrm{P}<0.05$; Fig. $7 \mathrm{~B})$. There was no significant difference in the level of p-Akt (T308) in the spinal cord between the high-n-3 PUFA diet group and the other two groups (Fig. 7B); however, the high-n-3 PUFA diet group had significantly higher levels of 4EBP1 activity in the spinal cord compared with the other two groups $(\mathrm{P}<0.05$; Fig. 7A and $\mathrm{B})$. Thus, a high n-3/n-6 PUFA diet could inhibit activity of the mTORC1 signaling pathway.

\section{Discussion}

In this study, we created a spinal cord contusion model in SD rats and fed them with a diet high in n-3 PUFAs to increase n-3 PUFA content as well as the n-3/n-6 PUFA composition; we investigated the effects of this change on recovery of SCI. As our results show, a diet high in n-3 PUFAs inhibited the mTORC1 signaling pathway, increased expression of autophagy proteins, provided energy for regeneration of the injured spinal cord and promoted the recovery of hind limb motor function.

Recently, a series of studies has demonstrated that n-3 PUFAs play crucial roles in tissue repair mechanisms (44-46), 
A

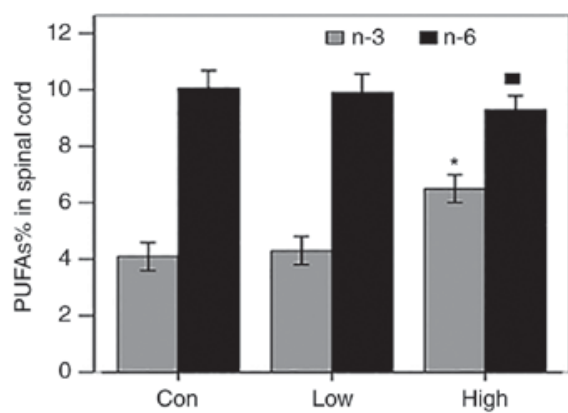

C

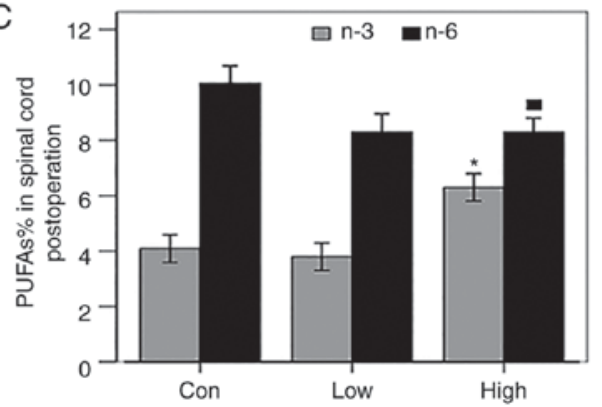

B
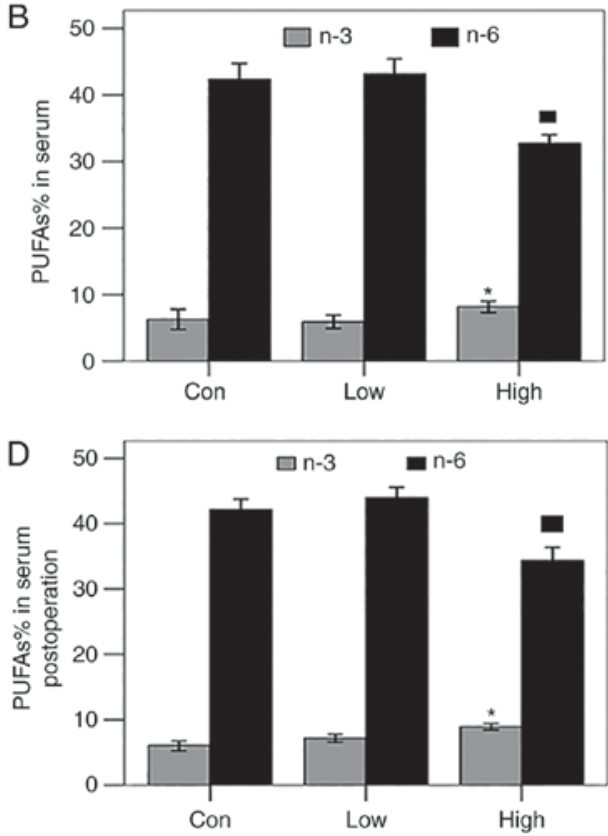

Figure 2. Effects of exogenous polyunsaturated fatty acids (PUFAs) on the percentage of n-3 PUFAs and n-6 PUFAs in the spinal cord and serum of each group. (A-D) The high-n-3 PUFA diet group showed a significantly higher content of n-3 PUFAs and lower n-6 PUFA content in the spinal cord and serum both preoperatively and postoperatively. ${ }^{*}$ Comparison with the low-n-3 PUFA diet group and the control group, $\mathrm{P}<0.05$.
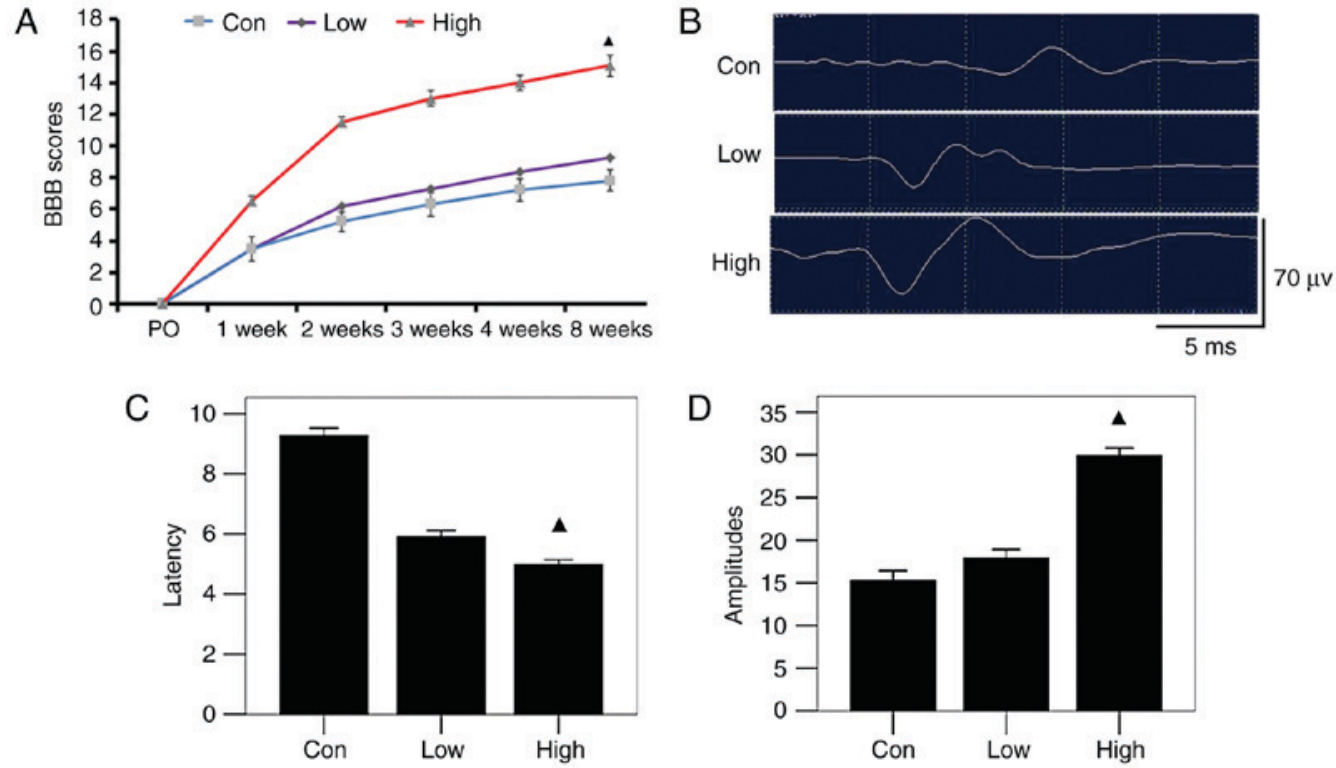

Figure 3. Motor function assessment of each group after operation. (A) Basso, Beattie and Bresnahan (BBB) scores at different time-points; the high-n-3 polyunsaturated fatty acid (PUFA) diet group had significantly higher scores at all time-points. (B) Electrophysiologic monitoring 8 weeks postoperatively. (C and D) As shown by latency time and amplitude of motor evoked potential, the high-n-3 PUFA diet group showed significantly lower latency time and higher amplitude. ${ }^{\wedge}$ Comparison with the low-n-3 PUFA diet group and the control group, $\mathrm{P}<0.05$.

so the study of the influence of n-3 PUFAs on physiological and pathological processes in the human body is very important (47-49). Traditional oral administration can change the content of n-3 PUFAs and the proportions of n-3/n- 6 PUFAs in the body; such administration has been used widely in the clinic and is much safer than transgenic technology $(13,15)$. Consequently, we supplied rats with n-3 PUFAs in their diet to try to reproduce drug delivery in the clinic through the enteral route and thus provide reference data for future clinical studies.
Better understanding of the effect of n-3 PUFAs on the mTORC1 signaling pathway in tissue repair will help greatly in increasing understanding of the repair of SCI and provide information which could lead to better treatments. A recent study reported that a diet high in n-3 PUFAs reduced expression of p-S6 and p-S6K, suggesting an inhibitory effect of n-3 PUFAs on the mTORC1 pathway (50). It has also been reported that both exogenous and endogenous n-3 PUFAs can target mTOR to inhibit the mTORC1/2 signaling pathway and the 
A
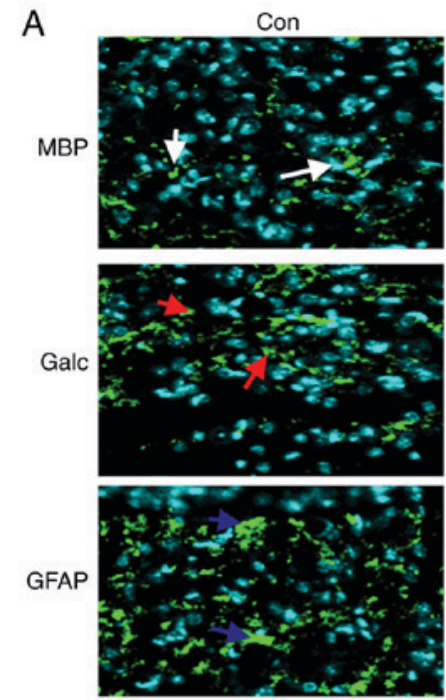
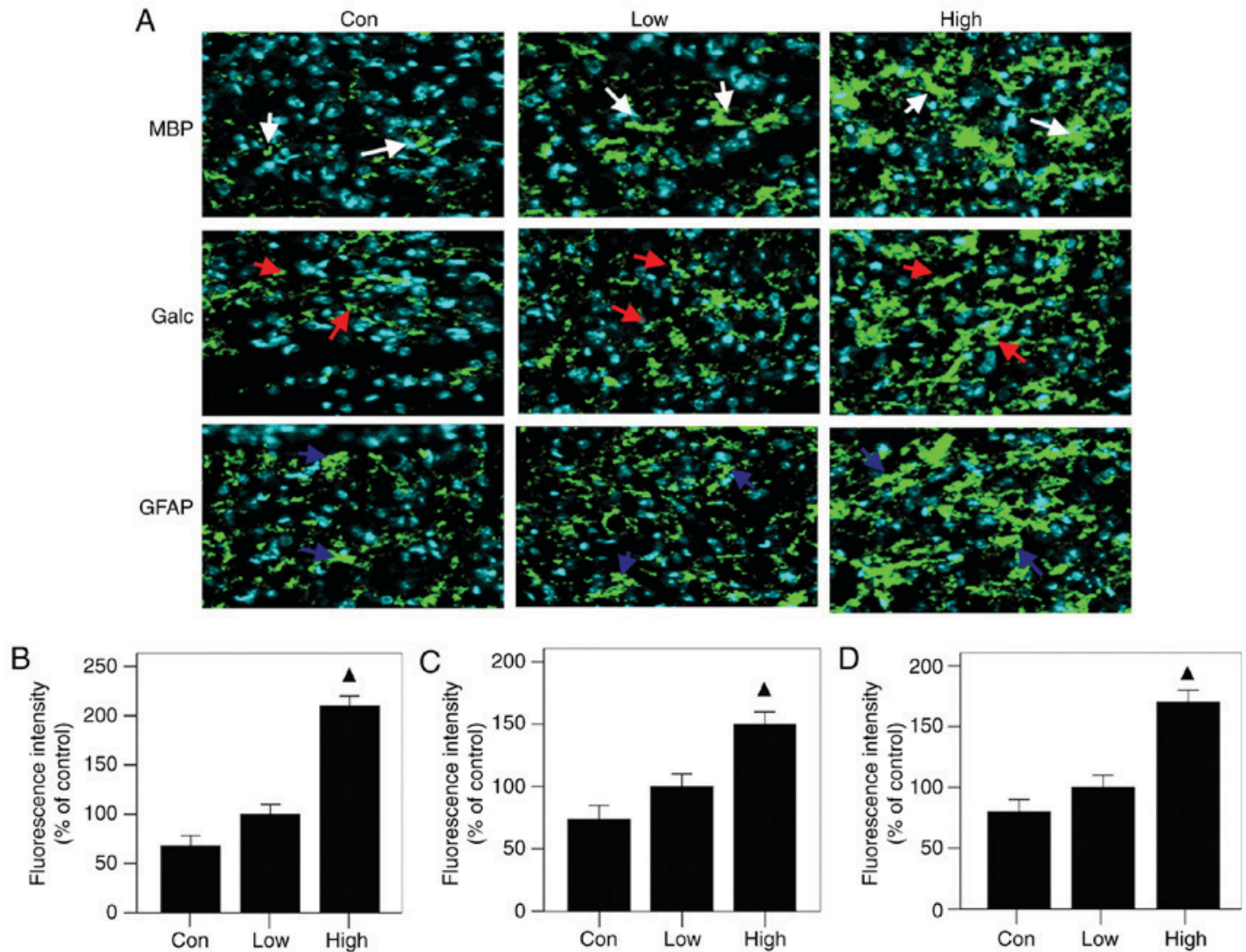

Figure 4. Relative tissue repair related proteins expression levels after operation. (A) Results of MBP, Galc and GFAP immunofluorescent staining (white arrows: MBP, red arrows: Galc, blue: GFAP). (B-D) Analysis of results: The levels of MBP, GFAP and Galc, in the high-n-3 PUFA diet group were all significantly higher. ${ }^{\mathbf{A}}$ Comparison with the low-n-3 polyunsaturated fatty acid (PUFA) diet group and the control group, $\mathrm{P}<0.05$.
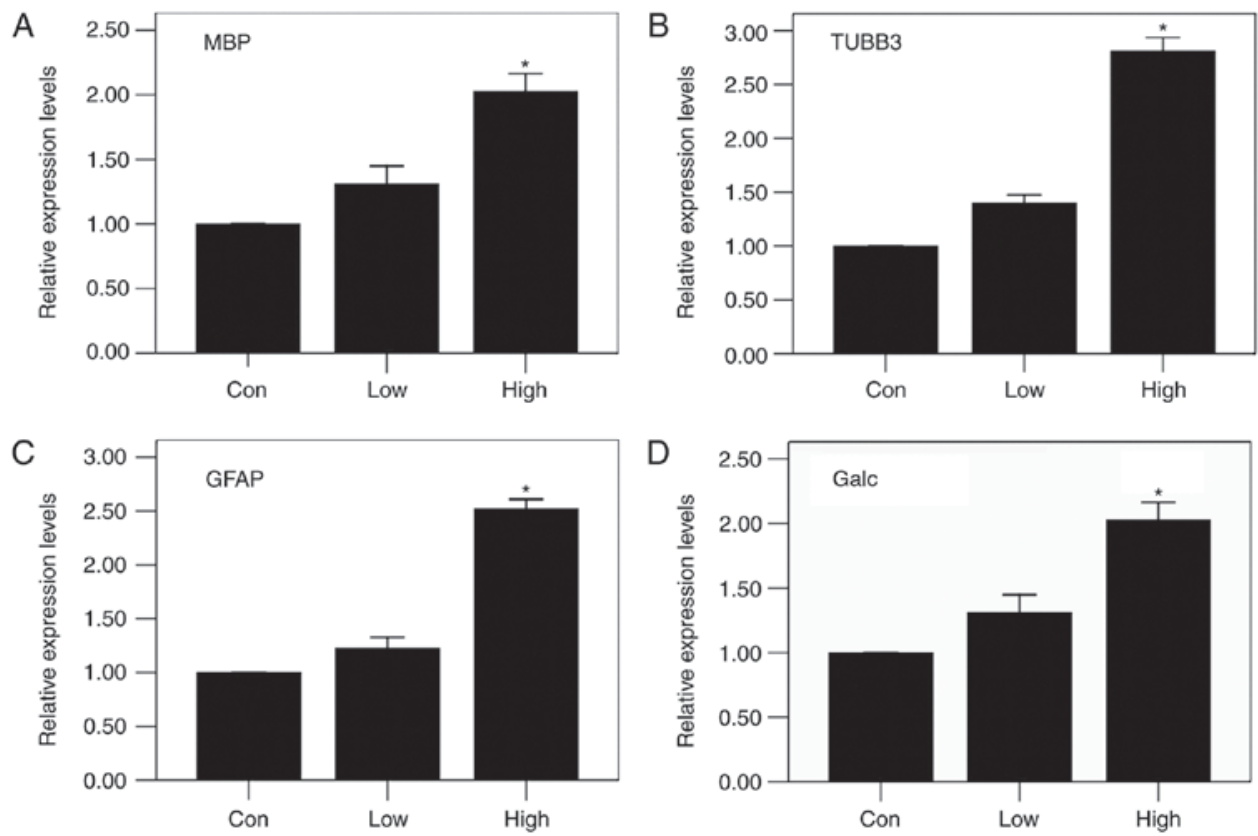

Figure 5. Relative mRNA expression levels after operation by RT-PCR. In the high-n-3 PUFA diet group, the levels of (A) MBP, (B) TUBB3, (C) GFAP and (D) Galc all increased significantly after surgery. ${ }^{*}$ Comparison with the low-n-3 polyunsaturated fatty acid (PUFA) diet group and the control group, $\mathrm{P}<0.05$.

downstream proteins (51). Our western blot results showed that expression of p-S6K, p-Akt, and 4-EBP1 in the injured spinal cord of rats fed a high-n-3 PUFA diet were significantly reduced by 8 weeks after injury, consistent with the above findings. As a result, we concluded that a diet high in n-3 PUFAs can inhibit activity of the mTOR signaling pathway in SCI.

After SCI, the local blood supply, and hence the supply of energy needed for physiological and pathological activities, is 
A
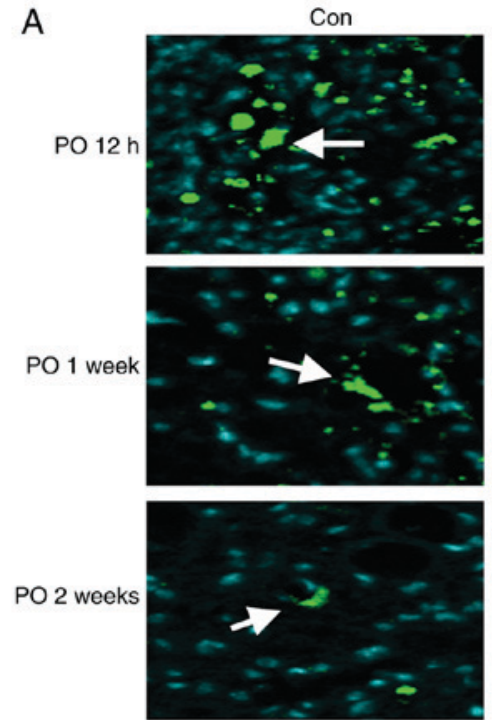
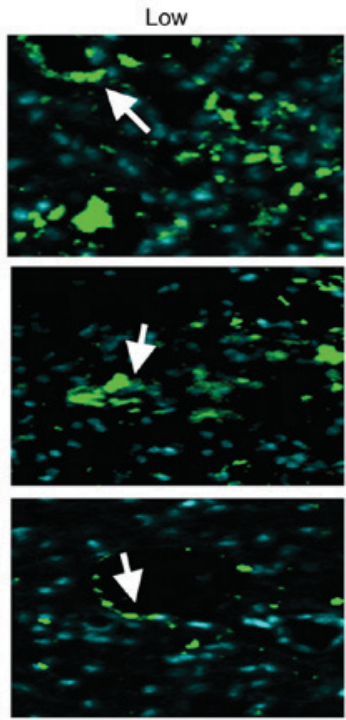

C

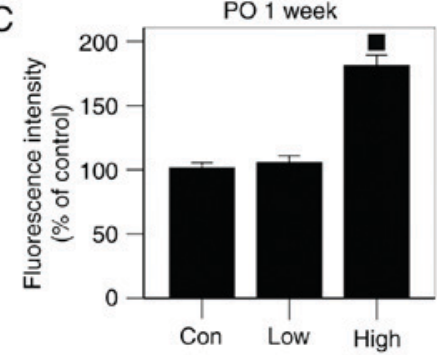

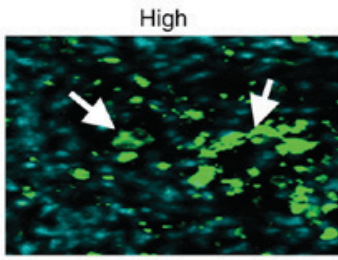
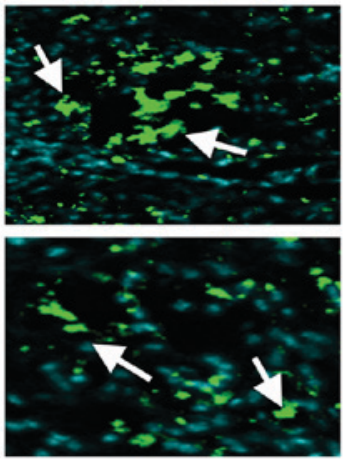

D

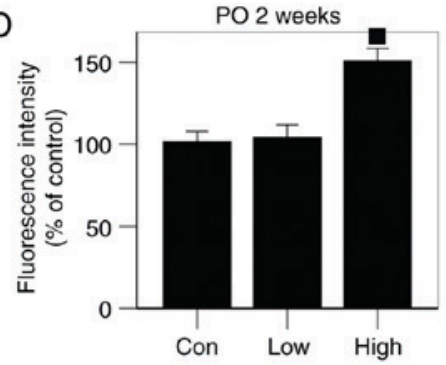

Figure 6. Expression levels of autophagy-related protein. (A) Results of immunofluorescent staining (white arrows: LC3II). (B-D) In the high-n-3 polyunsatu-

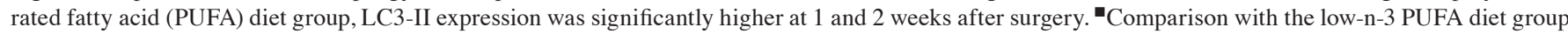
and the control group, $\mathrm{P}<0.05$.
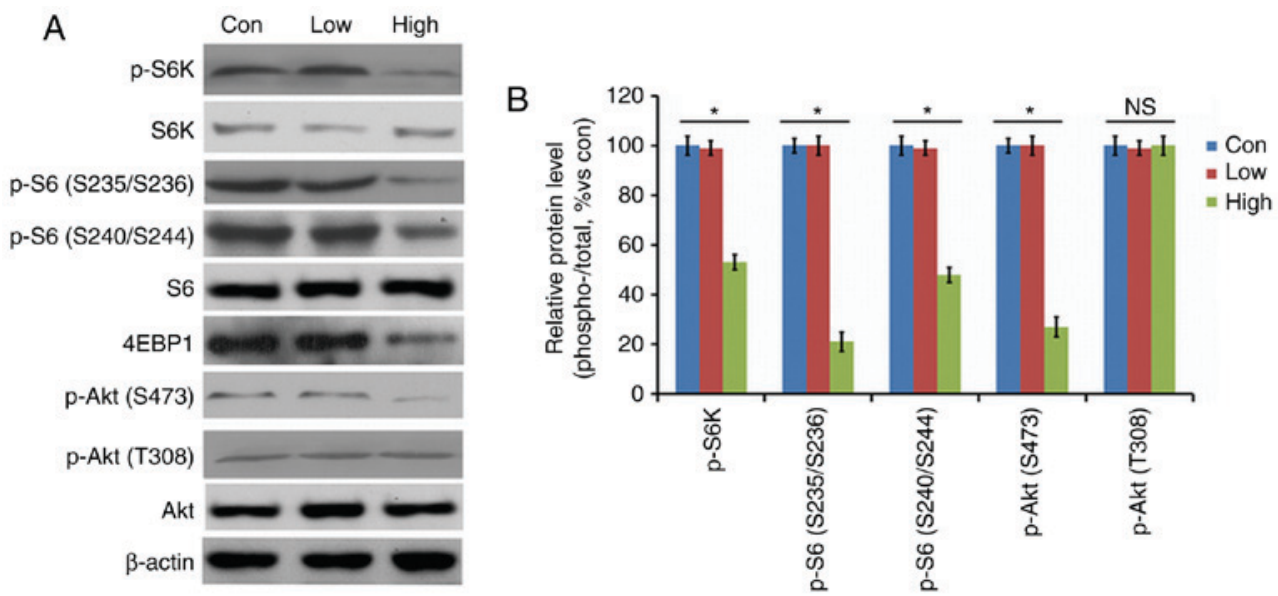

Figure 7. Expression levels of mammalian target of rapamycin (mTOR)-related proteins after operation. Results of (A) western blotting. (B) Activities of p-Akt (S473), p-S6 (S235/S236), p-S6 (S240/S244) and p-S6K were reduced significantly in the high-n-3 polyunsaturated fatty acid (PUFA) diet group, while the opposite effect was observed for P-Akt (T308). "Comparison with the low-n-3 PUFA diet group and the control group, $\mathrm{P}<0.05$.

damaged due to local vascular system damage and regeneration of the injured spinal cord is difficult. Many investigators have carried out preliminary research and found that autophagy is significantly activated in the days just after SCI in a contusion model, peaking within a week postoperatively and then decreasing to levels close to normal after 2 weeks $(38,52-55)$. In this experiment, expression of autophagy markers showed a trend consistent with reports in the existing literature, and mTORC1 activity in the injured spinal cord of rats fed a high-n-3 PUFA diet was obviously reduced (Fig. 4), while LC3-II protein expression was enhanced. Increased cell autophagy activity removes damaged tissue and provides a large amount of macromolecular material and energy for the repair of local injured tissue, thus promoting regeneration of the injured spinal cord and restoration of motor function after $\mathrm{SCI}$ in rats. 
In conclusion, this study demonstrates that a high-n-3 PUFA diet downregulates the activity of the mTORC1 signaling pathway, improves autophagy capability, and provides energy to promote repair of the injured spinal cord and restoration of motor function in a rat model of SCI. Thus, it can be seen that changes of n-3 PUFA content and n-3/n-6 PUFA ratio play an important role in motor function recovery of SCI rats. These results provide important reference data for the potential treatment of SCI by n-3 PUFAs.

\section{Acknowledgements}

The present study was supported by National Natural Sciences Foundation of China (grant no. 81560213), Chongqing Three Gorges Central Hospital PhD Research Startup Fund (grant no. 2017BSKYQDJJ02), Chongqing Postdoctoral Research Special Fund (grant no. Xm2017181), and North Sichuan Medical College and Chongqing Three Gorges Central Hospital Cooperation Fund (grant no. 2016XY01).

\section{References}

1. Schwab ME: Regenerative nerve fiber in the adult central nervous system. News Physiol Sci 13: 294-298, 1998.

2. Fry EJ: Central nervous system regeneration: Mission impossible? Clin Exp Pharmacol Physiol 28: 253-258, 2001.

3. Ritfeld GJ, Nandoe Tewarie RD, Vajn K, Rahiem ST, Hurtado A, Wendell DF, Roos RA and Oudega M: Bone marrow stromal cell-mediated tissue sparing enhances functional repair after spinal cord contusion in adult rats. Cell Transplant 21: 1561-1575, 2012.

4. Ribatti D, Conconi MT, Nico B, Baiguera S, Corsi P, Parnigotto PP and Nussdorfer GG: Angiogenic response induced by acellular brain scaffolds grafted onto the chick embryo chorioallantoic membrane. Brain Res 989: 9-15, 2003.

5. Johnson PJ, Tatara A, Shiu A and Sakiyama-Elbert SE: Controlled release of neurotrophin-3 and platelet-derived growth factor from fibrin scaffolds containing neural progenitor cells enhances survival and differentiation into neurons in a subacute model of SCI. Cell Transplant 19: 89-101, 2011.

6. Zurita M, Vaquero J, Oya S and Miguel M: Schwann cells induce neuronal differentiation of bone marrow stromal cells. Neuroreport 16: 505-508, 2005.

7. Liu GM, Luo YG, Li J and Xu K: Knockdown of Nogo gene by short hairpin RNA interference promotes functional recovery of spinal cord injury in a rat model. Mol Med Rep 13: 4431-4436, 2016.

8. Guo J, Li Y, Chen Z, He Z, Zhang B, Li Y, Hu J, Han M, Xu Y and Li Y: N-acetylcysteine treatment following spinal cord trauma reduces neural tissue damage and improves locomotor function in mice. Mol Med Rep 12: 37-44, 2015.

9. Chen KB, Uchida K, Nakajima H, Yayama T, Hirai T, Watanabe S, Guerrero AR, Kobayashi S, Ma WY, Liu SY and Baba H: Tumor necrosis factor- $\alpha$ antagonist reduces apoptosis of neurons and oligodendroglia in rat spinal cord injury. Spine (Phila Pa 1976) 36: 1350-1358, 2011.

10. Figueroa JD and De Leon M: Neurorestorative targets of dietary long-chain omega-3 fatty acids in neurological injury. Mol Neurobiol 50: 197-213, 2014.

11. Zeman M, Jirak R, Vecka M, Raboch J and Zak A: N-3 polyunsaturated fatty acids in psychiatric diseases: Mechanisms and clinical data. Neuro Endocrinol Lett 33: 736-748, 2012.

12. Hasadsri L, Wang BH, Lee JV, Erdman JW, Llano DA, Barbey AK, Wszalek T, Sharrock MF and Wang HJ: Omega-3 fatty acids as a putative treatment for traumatic brain injury. J Neurotrauma 30: 897-906, 2013.

13. Huang MJ, Wang L, Jin DD, Zhang ZM, Chen TY, Jia CH, Wang Y, Zhen XC, Huang B, Yan B, et al: Enhancement of the synthesis of $n-3$ PUFAs in fat- 1 transgenic mice inhibits mTORC1 signalling and delays surgically induced osteoarthritis in comparison with wild-type mice. Ann Rheum Dis 73 1719-1727, 2014.
14. Lim SN, Gladman SJ, Dyall SC, Patel U, Virani N, Kang JX, Priestley JV and Michael-Titus AT: Transgenic mice with high endogenous omega-3 fatty acids are protected from spinal cord injury. Neurobiol Dis 51: 104-112, 2013.

15. Wen ZH, Su YC, Lai PL, Zhang Y, Xu YF, Zhao A, Yao GY, Jia $\mathrm{CH}$, Lin J, Xu S, et al: Critical role of arachidonic acid activated mTOR signaling in breast carcinogenesis and angiogenesis. Oncogene 32: 160-170, 2013.

16. Gerber M: Omega-3 fatty acids and cancers: A systematic update review of epidemiological studies. Br J Nutr 107 (Suppl 2): S228-S239, 2012.

17. Fleming JC, Norenberg MD, Ramsay DA, Dekaban GA, Marcillo AE, Saenz AD, Pasquale-Styles M, Dietrich WD and Weaver LC: The cellular inflammatory response in human spinal cords after injury. Brain 129: 3249-3269, 2006.

18. Donnelly DJ and Popovich PG: Inflammation and its role in neuroprotection, axonal regeneration and functional recovery after spinal cord injury. Exp Neurol 209: 378-388, 2008.

19. Daniel J and Klionsky: The molecular machinery of autophagy: unanswered questions. J Cell Sci 118: 7-18, 2005.

20. Kanki T and Klionsky DJ: The molecular mechanism of mitochondria autophagy in yeast. Mol Microbiol 75: 795-800, 2010.

21. Kanno H, Ozawa H, Sekiguchi A, Yamaya S, Tateda S, Yahata K and Itoi E: The role of mTOR signaling pathway in spinal cord injury. Cell Cycle 11: 3175-3179, 2012.

22. Liu K, Lu Y,Lee JK, Samara R, Willenberg R, Sears-Kraxberger I, Tedeschi A, Park KK, Jin D, Cai B, et al: PTEN deletion enhances the regenerative ability of adult corticospinal neurons. Nat Neurosci 13: 1075-10781, 2010.

23. Lamming DW and Sabatini DM: A central role for mTOR in lipid homeostasis. Cell Metab 18: 465-469, 2013.

24. Laplante M and Sabatini DM: An emerging role of mTOR in lipid biosynthesis. Curr Biol 19: R1046-R1052, 2009.

25. Laplante M and Sabatini DM: mTOR signaling at a glance. J Cell Sci 122: 3589-3594, 2009.

26. Laplante M and Sabatini DM: mTOR signaling. Cold Spring Harb Perspect Biol 4: pii: a011593, 2012.

27. Su M, Guan H, Zhang F, Gao Y, Teng X and Yang W: HDAC6 regulates the chaperone-mediated autophagy to prevent oxidative damage in injured neurons after experimental spinal cord injury. Oxid Med Cell Longev 2016: 7263736, 2016.

28. Zhou KL, Zhou YF, Wu K, Tian NF, Wu YS, Wang YL, Chen DH, Zhou B, Wang XY, Xu HZ and Zhang XL: Stimulation of autophagy promotes functional recovery in diabetic rats with spinal cord injury. Sci Rep 5: 17130, 2015.

29. Zhou Y, Zheng B, Ye L, Zhang H, Zhu S, Zheng X, Xia Q, He Z, Wang Q, Xiao J and Xu H: Retinoic acid prevents disruption of blood-spinal cord barrier by inducing autophagic flux after spinal cord injury. Neurochem Res 41: 813-825, 2016.

30. Gao K, Wang G, Wang Y, Han D, Bi J, Yuan Y, Yao T, Wan Z, $\mathrm{Li} \mathrm{H}$ and Mei X: Neuroprotective effect of simvastatin via inducing the autophagy on spinal cord injury in the rat model. Biomed Res Int 2015: 260161, 2015.

31. Guo Y, Liu S, Zhang X, Wang L, Gao J, Han A and Hao A: G-CSF promotes autophagy and reduces neural tissue damage after spinal cord injury in mice. Lab Invest 95: 1439-1449, 2015.

32. Lotz MK and Caramés B: Autophagy and cartilage homeostasis mechanisms in joint health, aging and OA. Nat Rev Rheumatol 7: 579-587, 2011.

33. Kanno H, Ozawa H, Sekiguchi A, Yamaya S and Itoi E: Induction of autophagy and autophagic cell death in damaged neural tissue after acute spinal cord injury in mice. Spine (Phila Pa 1976) 36: E1427-E1434, 2011.

34. Basso DM, Beattie MS and Bresnahan JC: A sensitive and reliable locomotor rating scale for open field testing in rats. J Neurotrauma 12: 1-21, 1995.

35. Zhang XY, Xue H, Liu JM and Chen D: Chemically extracted acellular muscle: A new potential scaffold for spinal cord injury repair. J Biomed Mater Res A 100: 578-587, 2012.

36. Wei D, Li J, Shen M, Jia W, Chen N, Chen T, Su D, Tian H, Zheng S, Dai Y and Zhao A: Cellular production of n-3 PUFAs and reduction of n-6-to-n-3 ratios in the pancreatic beta-cells and islets enhance insulin secretion and confer protection against cytokine-induced cell death. Diabetes 59: 471-478, 2010.

37. Kang JX: A transgenic mouse model for gene-nutrient interactions. J Nutrigenet Nutrigenomics 1: 172-177, 2008. 
38. Nakamura M, Houghtling RA, MacArthur L, Bayer BM and Bregman BS: Differences in cytokine gene expression profile between acute and secondary injury in adult rat spinal cord. Exp Neurol 184: 313-325, 2003.

39. Manku MS, Horrobin DF, Huang YS and Morse N: Fatty acids in plasma and red cell membranes in normal humans. Lipids 18: 906-908, 1983

40. Dyall SC, Michael GJ, Whelpton R, Scott AG and Michael-Titus AT: Dietary enrichment with omega-3 polyunsaturated fatty acids reverses age-related decreases in the GluR2 and NR2B glutamate receptor subunits in rat forebrain. Neurobiol Aging 28: 424-439, 2007.

41. Jiang W, Zhu Z, McGinley JN, El Bayoumy K, Manni A and Thompson HJ: Identification of a molecular signature underlying inhibition of mammary carcinoma growth by dietary n-3 fatty acids. Cancer Res 72: 3795-3806, 2012.

42. Chen Z, Zhang Y, Jia C, Wang Y, Lai P, Zhou X, Wang Y, Song Q, Lin J, Ren Z, et al: mTORC1/2 targeted by $n-3$ polyunsaturated fatty acids in the prevention of mammary tumorigenesis and tumor progression. Oncogene 33: 4548-4557, 2014

43. Berquin IM, Edwards IJ and Chen YQ: Multi-targeted therapy of cancer by omega-3 fatty acids. Cancer Lett 269: 363-377, 2008.

44. Larsson SC, Kumlin M, Ingelman-Sundberg M and Wolk A: Dietary long-chain n-3 fatty acids for the prevention of cancer: A review of potential mechanisms. Am J Clin Nutr 79: 935-945, 2004.

45. Streit WJ, Semple-Rowland SL, Hurley SD, Miller RC, Popovich PG and Stokes BT: Cytokine mRNA profiles in contused spinal cord and axotomized facial nucleus suggest a beneficial role for inflammation and gliosis. Exp Neurol 152: 74-87, 1998

46. Bracken MB, Shepard MJ, Collins WF, Holford TR, Young W, Baskin DS, Eisenberg HM, Flamm E, Leo-Summers L, Maroon J, et al: A randomized, controlled trial of methylprednisolone or naloxone in the treatment of acute spinal-cord injury. Results of the second national acute spinal cord injury study. N Engl J Med 322: 1405-1411, 1990.

47. Wang CX, Olschowka JA and Wrathall JR: Increase of interleukin-lbeta mRNA and protein in the spinal cord following experimental traumatic injury in the rat. Brain Res 13: 190-196, 1997.
48. Lu Y, Jiang BC, Cao DL, Zhang ZJ, Zhang X, Ji RR and Gao YJ: TRAF6 upregulation in spinal astrocytes maintains neuropathic pain by integrating TNF- $\alpha$ and IL-1 $\beta$ signaling. Pain 155 2618-2629, 2014

49. Grau JW, Huie JR, Lee KH, Hoy KC, Huang YJ, Turtle JD, Strain MM, Baumbauer KM, Miranda RM, Hook MA, et al: Metaplasticity and behavior: How training and inflammation affect plastic potential within the spinal cord and recovery after injury. Front Neural Circuits 8: 100, 2014.

50. Erlich S, Alexandrovich A, Shohami E and Pinkas-Kramarski R: Rapamycin is a neuroprotective treatment for traumatic brain injury. Neurobiol Dis 26: 86-93, 2007.

51. Hou H, Zhang L, Zhang L and Tang P: Acute spinal cord injury in rats should target activated autophagy. J Neurosurg Spine 20: 568-577, 2014.

52. Hao HH, Wang L, Guo ZJ, Bai L, Zhang RP, Shuang WB, Jia YJ, Wang J, Li XY and Liu Q: Valproic acid reduces autophagy and promotes functional recovery after spinal cord injury in rats. Neurosci Bull 29: 484-492, 2013.

53. Wang ZY, Lin JH, Muharram A and Liu WG: Beclin-1-mediated autophagy protects spinal cord neurons against mechanical injury-induced apoptosis. Apoptosis 19: 933-945, 2014.

54. Serhan CN, Dalli J, Colas RA, Winkler JW and Chiang N: Protectins and maresins: New pro-resolving families of mediators in acute inflammation and resolution bioactive metabolome. Biochim Biophys Acta 1851: 397-413, 2015.

55. Chen Z, Fu Q, Shen B, Huang X, Wang K, He P, Li F, Zhang F and Shen H: Enhanced p62 expression triggers concomitant autophagy and apoptosis in a rat chronic spinal cord compression model. Mol Med Rep 9: 2091-2096, 2014.

(i) This work is licensed under a Creative Commons

c. International (CC BY-NC-ND 4.0) License. 